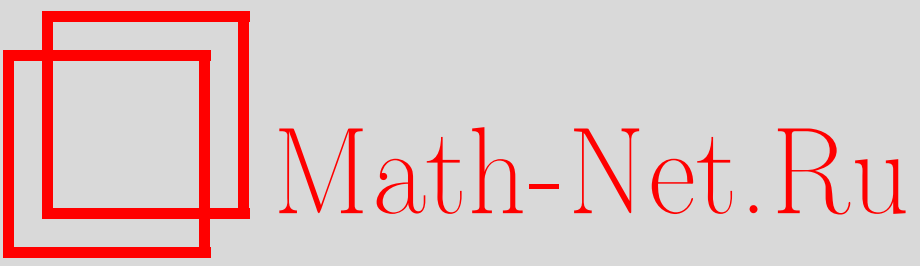

И. В. Денисов, А. И. Денисов, Математические модели процессов горения, Итоги науки и техн. Сер. Соврем. мат. и ее прил. Темат. обз., 2020, том 185, 50-57

DOI: https://doi.org/10.36535/0233-6723-2020-185-50-57

Использование Общероссийского математического портала Math-Net.Ru подразумевает, что вы прочитали и согласны с пользовательским соглашением

http://www.mathnet.ru/rus/agreement

Параметры загрузки:

IP: 54.224 .60 .19

26 апреля 2023 г., 15:20:40 


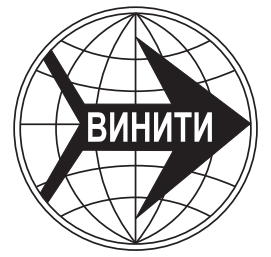

ИТОГИ НАУКИ И ТЕХНИКИ.

Современная математика и ее приложения.

Тематические обзоры.

Том 185 (2020). C. $50-57$

DOI: $10.36535 / 0233-6723-2020-185-50-57$

УДК 517.956 .4

\title{
МАТЕМАТИЧЕСКИЕ МОДЕЛИ ПРОЦЕССОВ ГОРЕНИЯ
}

\author{
(c) 2020 г. И. В. ДЕНИСОВ, А. И. ДЕНИСОВ
}

\begin{abstract}
АннотАция. Рассматриваются модельные задачи химической кинетики, которые приводят к сингулярно возмущенным параболическим уравнениям в областях с негладкими границами. Разрешимость таких задач зависит от свойств неоднородности в угловых точках границы.
\end{abstract}

Ключевъе слова: пограничный слой, сингулярно возмущенное уравнение, асимптотическое приближение.

\section{MATHEMATICAL MODELS OF COMBUSTION PROCESSES}

\author{
(c) 2020 I. V. DENISOV, A. I. DENISOV
}

\begin{abstract}
In this paper, we consider model problems of chemical kinetics that lead to singularly perturbed parabolic equations in domains with nonsmooth boundaries. The solvability of such problems depends on the properties of the inhomogeneity at corner points of the boundary.
\end{abstract}

Keywords and phrases: boundary layer, singularly perturbed equation, asymptotic approximation.

AMS Subject Classification: 34E10

1. Введение. Задачи химической кинетики с учетом диффузии (в частности, процессы горения веществ) приводят к системам сингулярно возмущенных параболических уравнений

$$
\begin{aligned}
& \varepsilon\left(\frac{\partial u}{\partial t}-a \Delta u\right)=f(u, v, x, t, \varepsilon), \\
& \frac{\partial v}{\partial t}-b \Delta v=g(u, v, x, t, \varepsilon),
\end{aligned}
$$

с трехмерной переменной $x=\left(x_{1}, x_{2}, x_{3}\right)$. Векторные функции $u$ и $v$ составляются из концентраций реагирующих веществ. Параметр $\varepsilon$ соответствует величине, получающейся делением на скорости быстрых реакций, и считается малым. Обычно рассматривают только уравнение с быстрыми переменными, а в качестве $x$ берут одномерную переменную. Для удобства вычислений малый параметр обозначают как $\varepsilon^{2}$. Если уравнение (1) является линейным и рассматривается в области с гладкими границами, то асимптотическое разложение решения получается методом Люстерника-Вишика [3].

Если границы области не являются гладкими, то асимптотическое разложение решения получается методом угловых пограничных функций В. Ф. Бутузова (см. [1]). Для нелинейных сингулярно возмущенных параболических уравнений с краевыми условиями первого рода в областях с негладкими границами первые результаты получены в [4]. В этой работе было рассмотрено уравнение с функцией $f$, являющейся квадратичной и монотонной на промежутке от корня вырожденного уравнения до граничного значения. При построении асимптотики решения пришлось доказывать разрешимость нелинейных параболических задач, аналогичных исходной. Однако ограничениям, поставленным в работе, удовлетворяли не все квадратичные функции. В [5] метод был распространен на все квадратичные функции. 
2. Постановка задачи. Рассмотрим сингулярно возмущенное параболическое уравнение

$$
\varepsilon^{2}\left(a^{2} \frac{\partial^{2} u}{\partial x^{2}}-\frac{\partial u}{\partial t}\right)=F(u, x, t, \varepsilon), \quad(x, t) \in \Omega,
$$

где $\Omega:=\{(x, t) \mid 0<x<1,0<t<T\}-$ прямоугольник. Потребуем выполнение начального условия

$$
u(x, 0, \varepsilon)=\phi(x), \quad 0 \leqslant x \leqslant 1,
$$

и краевых условий первого рода

$$
u(0, t, \varepsilon)=\psi_{1}(t), u(1, t, \varepsilon)=\psi_{2}(t), \quad 0 \leqslant t \leqslant T .
$$

Пусть выполнены следующие условия.

I. Функции $F(u, x, t, \varepsilon), \phi(x), \psi_{1}(t)$ и $\psi_{2}(t)$ являются достаточно гладкими и в угловых точках прямоугольника $\Omega$ выполняются условия согласованности начально-краевых значений:

$$
\phi(0)=\psi_{1}(0), \quad \phi(1)=\psi_{2}(0) .
$$

II. Уравнение $F(u, x, t, 0)=0$ в замкнутом прямоугольнике $\bar{\Omega}$ имеет решение $u=\bar{u}_{0}(x, t)$. (Заметим, что в силу нелинейности это уравнение может иметь и другие решения.)

III. Производная $F_{u}^{\prime}\left(\bar{u}_{0}(x, t), x, t, 0\right)$ положительна в замкнутом прямоугольнике $\bar{\Omega}$.

IV. Начальная задача

$$
\frac{d \Pi_{0}}{d \tau}=-F\left(\bar{u}_{0}(x, 0)+\Pi_{0}, x, 0,0\right), \quad \tau \geqslant 0, \quad \Pi_{0}(x, 0)=\phi(x)-\bar{u}_{0}(x, 0),
$$

имеет решение $\Pi_{0}(x, \tau)$, удовлетворяющее условию $\Pi_{0}(x, \infty)=0$.

V. Для систем

$$
\frac{d z_{1}}{d y}=z_{2}, \quad a^{2} \frac{d z_{2}}{d y}=F\left(\bar{u}_{0}(k, t)+z_{1}, k, t, 0\right),
$$

прямые $z_{1}=\psi_{1+k}(t)-\bar{u}_{0}(k, t)$ пересекают сепаратрисы, входящие в точку покоя $\left(z_{1}, z_{2}\right)=$ $(0,0)$ при $y \rightarrow \infty$ (здесь $t$ - параметр, $k=0$ или 1 ).

Условий I-V недостаточно, чтобы гарантировать существование решения задачи (3)-(5). Поэтому требуются дополнительные условия, гарантирующие возможность построения асимптотики решения.

Решение задачи (3)-(5) строится согласно методу угловых пограничных функций в виде суммы

$$
u(x, t, \varepsilon)=\bar{u}+\Pi+Q+Q^{*}+P+P^{*},
$$

где $\bar{u}$ - функция, называемая регулярной частью асимптотики. Эта функция представляет решение задачи во внутренней части прямоугольника $\Omega$ без учета граничных условий. Пограничные функции $\Pi, Q$ и $Q^{*}$ осуществляют гладкий переход от регулярной части к граничным условиям на сторонах $t=0, x=0$ и $x=1$ прямоугольника $\Omega$ соответственно. Угловые пограничные функции $P$ и $P^{*}$ сглаживают невязки, вносимые пограничными функциями вблизи вершин $(0,0)$ и $(1,0)$ прямоугольника $\Omega$ соответственно.

3. Регулярная и погранслойная части асимптотики. Формальная процедура построения регулярной части асимптотики и погранслойных функций хорошо отработана (см. [2]), и мы повторим ее схематично. В уравнении (3) функцию $F$ заменим выражением, аналогичным (9):

$$
F(u, x, t, \varepsilon)=\bar{F}+\left(\Pi F+Q F+Q^{*} F\right)+\left(P F+P^{*} F\right) .
$$

Выражения (9) и (10) подставим в уравнение (3) и разделим его на шесть частей: регулярную, три погранслойных и две угловых. Регулярная часть асимптотики $\bar{u}$ строится в виде ряда по степеням $\varepsilon$ :

$$
\bar{u}(x, t, \varepsilon)=\sum_{k=0}^{\infty} \varepsilon^{k} \bar{u}_{k}(x, t) .
$$

Коэффициент $\bar{u}_{0}=\bar{u}_{0}(x, t)$ выбирается в соответствии с условиями II и III, а последующие функции $\bar{u}_{k}, k \geqslant 1$, строятся рекуррентно. 
Пусть $\partial \Omega$ - граница прямоугольника $\Omega$ без стороны $t=T$. Регулярная часть $\bar{u}(x, t, \varepsilon)$ асимптотики дает решение задачи (3)-(5) внутри прямоугольника $\Omega$, но на границе $\partial \Omega$ функция $\bar{u}(x, t, \varepsilon)$, вообще говоря, не совпадает с начальным и граничными значениями. В связи с этим возникает так называемая «невязка».

Погранслойная часть асимптотики вводится для устранения невязок регулярной части с начальным и граничными условиями. Погранслойные функции $\Pi, Q$ и $Q^{*}$ определяются из уравнений, в которых произведен переход к растянутым переменным:

Функции $П, Q$ и $Q^{*}$ ищем в виде рядов

$$
\xi=\frac{x}{\varepsilon}, \quad \xi_{*}=\frac{1-x}{\varepsilon}, \quad \tau=\frac{t}{\varepsilon^{2}} .
$$

$$
\begin{aligned}
\Pi(x, \tau, \varepsilon) & =\sum_{k=0}^{\infty} \varepsilon^{k} \Pi_{k}(x, \tau), \\
Q(\xi, t, \varepsilon) & =\sum_{k=0}^{\infty} \varepsilon^{k} Q_{k}(\xi, t), \\
Q^{*}\left(\xi_{*}, t, \varepsilon\right) & =\sum_{k=0}^{\infty} \varepsilon^{k} Q_{k}^{*}\left(\xi_{*}, t\right) .
\end{aligned}
$$

На стороне $t=0$ невязка в начальном условии (4) призвана устранить функция $\Pi=\Pi(x, \tau, \varepsilon)$. При переходе от переменных $(x, t)$ к переменным $(x, \tau)$ прямоугольник $\Omega$ при $\varepsilon \rightarrow 0$ растягивается до полуполосы $0 \leqslant x \leqslant 1,0 \leqslant \tau<\infty$.

Для $\Pi_{0}=\Pi_{0}(x, \tau)$ получается задача

$$
-\frac{\partial \Pi_{0}}{\partial \tau}=F\left(\bar{u}_{0}(x, 0)+\Pi_{0}, x, 0,0\right), \quad \Pi_{0}(x, 0)=\phi(x)-\bar{u}_{0}(x, 0) .
$$

Здесь $x$ играет роль параметра. В силу условия IV эта задача имеет решение, для которого в силу условия III справедлива экспоненциальная оценка убывания вида

$$
\left|\Pi_{0}(x, \tau)\right| \leqslant C \exp (-\kappa \tau)
$$

где $C$ и $\kappa$-некоторые положительные числа.

Задачи для определения функций $\Pi_{k}=\Pi_{k}(x, \tau), k \geqslant 1$, получаются линейными:

$$
-\frac{\partial \Pi_{k}}{\partial \tau}=F_{u}^{\prime}\left(\bar{u}_{0}(x, 0)+\Pi_{0}, x, 0,0\right) \Pi_{k}+\tilde{\pi}_{k}, \quad \Pi_{k}(x, 0)=-\bar{u}_{k}(x, 0) .
$$

Функции $\tilde{\pi}_{k}$ представляют собой линейные комбинации функций $\Pi_{j}, j<k$, и их производных с коэффициентами, являющимися многочленами от $\tau$. Поэтому, если для функций $\Pi_{j}, j<k$, справедливы оценки вида (16), то для функций $\tilde{\pi}_{k}$ справедливы оценки того же вида.

Если величина $\phi(x)-\bar{u}_{0}(x, 0)$ не равна тождественно нулю, то решения задач $(17)$ имеют вид

$$
\Pi_{k}(x, \tau)=-U(x, \tau) \bar{u}_{k}(x, 0)-U(x, \tau) \int_{0}^{\tau}(U(x, \sigma))^{-1} \tilde{\pi}_{k}(x, \sigma) d \sigma,
$$

где

$$
U(x, \tau)=\exp \left(-\int_{0}^{\tau} F_{u}^{\prime}\left(\bar{u}_{0}(x, 0)+\Pi_{0}(x, \lambda), x, 0,0\right) d \lambda\right)
$$

- фундаментальное решение $(U(x, 0)=1)$ соответствующего однородного уравнения, и справедлива оценка вида

$$
\left|U(x, \tau)(U(x, \sigma))^{-1}\right| \leqslant C \exp (-\kappa(\tau-\sigma)),
$$

где $0 \leqslant x \leqslant 1,0 \leqslant \sigma \leqslant \tau$, а постоянные $C$ и $\kappa$-положительные числа. Эта оценка позволяет для функции $\Pi_{k}(x, \tau)$ получить оценку вида (16).

Если величина $\phi(x)-\bar{u}_{0}(x, 0) \equiv 0$, то $\Pi_{0}(x, \tau) \equiv 0$. Коэффициенты при $\Pi_{k}$ в задачах $(17)$ оказываются постоянными и положительными, т.е. задачи упрощаются. 
Таким образом определяются коэффициенты ряда $(12)$, и функция $\Pi(x, \tau, \varepsilon)$ устраняет невязки с начальным условием (4) на стороне $t=0$.

Построенная регулярная часть асимптотики вносит невязки и в граничные условия. На стороне $x=0$ невязки в граничных условиях призвана устранить функция $Q=Q(\xi, t, \varepsilon)$, где $\xi=x / \varepsilon-$ растянутая переменная. При переходе от переменных $(x, t)$ к переменным $(\xi, t)$ прямоугольник $\Omega$ при $\varepsilon \rightarrow 0$ растягивается до полуполосы $0 \leqslant \xi<\infty, 0 \leqslant t \leqslant T$.

Задача для $Q_{0}=Q_{0}(\xi, t)$ имеет вид

$$
a^{2} \frac{\partial^{2} Q_{0}}{\partial \xi^{2}}=F\left(\bar{u}_{0}(0, t)+Q_{0}, 0, t, 0\right), \quad Q_{0}(0, t)=\psi_{1}(t)-\bar{u}_{0}(0, t), \quad Q_{0}(\infty, t)=0 .
$$

где $t$ играет роль параметра. Уравнение (18) эквивалентно системе (8), в которой следует положить $z_{1}=Q_{0}(\xi, t), k=0, y=\xi$. Условия затухания выделяют решения уравнения (18), для которых справедливы экспоненциальные оценки убывания вида

$$
\left|Q_{0}(\xi, t)\right| \leqslant C \exp (-\kappa \xi)
$$

где $C$ и $\kappa$-положительные числа. Так как возможен переход с сепаратрисы на сепаратрису, то решение задачи (18) не единственно. Однако такие случаи мы исключаем и рассматриваем только монотонные решения.

Задачи для определения функций $Q_{k}(\xi, t), k \geqslant 1$, линейны:

$$
a^{2} \frac{\partial^{2} Q_{k}}{\partial \xi^{2}}=F_{u}^{\prime}\left(\bar{u}_{0}(0, t)+Q_{0}, 0, t, 0\right) Q_{k}+\tilde{q}_{k}, \quad Q_{k}(0, t)=-\bar{u}_{k}(0, t), \quad Q_{k}(\infty, t)=0 .
$$

Функции $\tilde{q}_{k}$ представляют собой линейные комбинации функций $Q_{j}, j<k$, и их производных с коэффициентами, являющимися многочленами от $\xi$. Поэтому, если для функций $Q_{j}, j<k$, справедливы экспоненциальные оценки вида (19), то для функций $\tilde{q}_{k}$ справедливы оценки того же вида.

Если величина $\psi_{1}(t)-\bar{u}_{0}(0, t)$ не равна тождественно нулю, то решение задачи $(20)$ имеет вид

$$
Q_{k}(\xi, t)=-\frac{\Phi(\xi, t)}{\Phi(0, t)} \bar{u}_{k}(0, t)-\frac{\Phi(\xi, t)}{a(0, t)} \int_{0}^{\xi} \frac{d \lambda}{\Phi^{2}(\lambda, t)} \int_{\lambda}^{\infty} \Phi(\sigma, t) \tilde{q}_{k}(\sigma, t) d \sigma, \quad \Phi(\xi, t)=\frac{\partial Q_{0}(\xi, t)}{\partial \xi}
$$

и для него справедлива оценка вида (19).

Если величина $\psi_{1}(t)-\bar{u}_{0}(0, t) \equiv 0$, то $Q_{0}(\xi, t) \equiv 0$, а коэффициент при $Q_{k}$ в уравнениях $(20)$ оказывается постоянным и положительным, т.е. задача упрощается.

Таким образом определяются коэффициенты ряда $(13)$, и функция $Q(\xi, t, \varepsilon)$ устраняет невязки в граничном условии на стороне $x=0$.

Регулярная часть асимптотики вносит невязки в граничные условия и на стороне $x=1$. Эти невязки устраняет функция $Q^{*}=Q^{*}\left(\xi_{*}, t, \varepsilon\right), \xi_{*}=(1-x) / \varepsilon$, которая строится в виде ряда (14). Коэффициенты этого ряда $Q_{k}^{*}$ определяются аналогично коэффициентам ряда $(13)$, и для них справедливы экспоненциальные оценки убывания вида

$$
\left|Q_{k}^{*}\left(\xi_{*}, t\right)\right| \leqslant C \exp \left(-\kappa \xi_{*}\right)
$$

где $C$ и $\kappa$-положительные числа.

Таким образом, погранслойная часть асимптотики определяется полностью. Однако каждая в отдельности погранслойная функция, устраняя невязки на соответствующей стороне, в свою очередь вносит невязки на примыкающие стороны прямоугольника. Так, погранслойные функции $\Pi_{k}(x, \tau)$, устраняя невязки в начальном условии на стороне $t=0$, вносят дополнительные невязки в граничные условия на сторонах $x=0$ и $x=1$. Эти невязки существенны только вблизи угловых точек $(0,0)$ и $(1,0)$, а далее, с ростом $t$, они экспоненциально затухают. Аналогичное влияние функции $Q_{k}(\xi, t)$ и $Q_{k}^{*}\left(\xi_{*}, t\right)$ оказывают на начальное условие на стороне $x=0$. 
4. Угловая часть асимптотики. Основные проблемы. С целью устранения невязок с начальным и граничными условиями вблизи угловых точек $(0,0)$ и $(1,0)$ прямоугольника $\Omega$ вводятся угловые пограничные функции $P(\xi, \tau, \varepsilon)$ и $P^{*}\left(\xi_{*}, \tau, \varepsilon\right)$. Ввиду того, что не существует универсального метода их нахождения, задачи для определения функций $P(\xi, \tau, \varepsilon)$ и $P^{*}\left(\xi_{*}, \tau, \varepsilon\right)$ доставляют основные трудности. Практически каждая такая задача требует разработки новых методов решения.

Угловые пограничные функции будем искать в виде рядов

$$
P(\xi, \tau, \varepsilon)=\sum_{k=0}^{\infty} \varepsilon^{k} P_{k}(\xi, \tau), \quad P^{*}\left(\xi_{*}, \tau, \varepsilon\right)=\sum_{k=0}^{\infty} \varepsilon^{k} P_{k}^{*}\left(\xi_{*}, \tau\right) .
$$

Рассмотрим угловую точку $(0,0)$. В окрестности этой точки невязки в условия $(4),(5)$ вносят функции $\Pi(x, \tau, \varepsilon)$ и $Q(\xi, t, \varepsilon)$. Для устранения этих невязок служит функция $P$, которая определяется из уравнения

$$
\left.\varepsilon^{2}\left(a^{2} \frac{\partial^{2} P}{\partial x^{2}}-\frac{\partial P}{\partial t}\right)\right|_{\substack{x=\varepsilon \xi \\ t=\varepsilon^{2}}}=P F .
$$

Задача для определения $P_{0}(\xi, \tau)$ ставится в первой четверти $\mathbb{R}_{+}^{2}=\{(\xi, \tau) \mid \xi>0, \tau>0\}$ плоскости переменных $(\xi, \tau)$ и имеет вид

$$
\begin{gathered}
a^{2} \frac{\partial^{2} P_{0}}{\partial \xi^{2}}-\frac{\partial P_{0}}{\partial \tau}=F\left(\bar{u}_{0}(0,0)+\Pi_{0}(0, \tau)+Q_{0}(\xi, 0)+P_{0}(\xi, \tau), 0,0,0\right)- \\
-F\left(\bar{u}_{0}(0,0)+\Pi_{0}(0, \tau), 0,0,0\right)-F\left(\bar{u}_{0}(0,0)+Q_{0}(\xi, 0), 0,0,0\right), \quad \xi>0, \tau>0, \\
P_{0}(0, \tau)=-\Pi_{0}(0, \tau), \quad P_{0}(\xi, 0)=-Q_{0}(\xi, 0), \quad P_{0}(\xi, \tau) \rightarrow 0 \text { при } \xi+\tau \rightarrow \infty .
\end{gathered}
$$

Для функций $P_{k}(\xi, \tau), k \geqslant 1$, в области $\mathbb{R}_{+}^{2}$ получаются линейные задачи

$$
\begin{aligned}
& a^{2} \frac{\partial^{2} P_{k}}{\partial \xi^{2}}-\frac{\partial P_{k}}{\partial \tau}=F_{u}^{\prime}\left(\bar{u}_{0}(0,0)+\Pi_{0}(0, \tau)+Q_{0}(\xi, 0)+P_{0}(\xi, \tau), 0,0,0\right) P_{k}+h_{k}, \\
& P_{k}(0, \tau)=-\Pi_{k}(0, \tau), \quad P_{k}(\xi, 0)=-Q_{k}(\xi, 0), \quad P_{k}(\xi, \tau) \rightarrow 0 \text { при } \xi+\tau \rightarrow \infty,
\end{aligned}
$$

где неоднородности $h_{k}$ удовлетворяют экспоненциальным оценкам вида

$$
\left|h_{k}(\xi, \tau)\right| \leqslant C \exp (-\kappa(\xi+\tau)),
$$

если такого же вида оценкам удовлетворяют функции $P_{0}, \ldots, P_{k-1}$. Здесь $C$ и $\kappa$-некоторые положительные числа.

Если $\phi(0,0)-\bar{u}_{0}(0,0)=0$, то решением задачи $(15)$ при $x=0$ будет функция $\Pi_{0}(0, \tau) \equiv 0$, решением задачи (17) при $t=0$ будет функция $Q_{0}(\xi, 0) \equiv 0$. Решением задачи $(21)$, (22) будет функция $P_{0}(\xi, \tau) \equiv 0$, а коэффициент в задачах $(23),(24)$ будет постоянным и положительным:

$$
F_{u}^{\prime}\left(\bar{u}_{0}(0,0)+\Pi_{0}(0, \tau)+Q_{0}(\xi, 0)+P_{0}(\xi, \tau), 0,0,0\right)=F_{u}^{\prime}\left(\bar{u}_{0}\right)>0 .
$$

В этом случае решения задач (23), (24) выписываются в явном виде, и для них получаются экспоненциальные оценки вида (25).

Если $\phi(0,0)-\bar{u}_{0}(0,0) \neq 0$, то, вообще говоря, не известно, имеет или нет задача $(21),(22)$ решение и удовлетворяет ли решение в случае существования экспоненциальной оценке вида (25). Кроме этого, в задачах $(23),(24)$ коэффициент $F_{u}^{\prime}\left(\bar{u}_{0}\right)$ может в зависимости от вида функции $F$ и величины $\phi(0,0)$ принимать как положительные, так и отрицательные значения.

Даже в случае разрешимости задач (21)-(24) доказательство того, что задача (3)-(5) имеет решение, все равно остается проблемой. Это связано с тем, что, не зная явного вида функции $P_{0}(\xi, \tau)$, мы не можем знать явного вида коэффициента $F_{u}^{\prime}\left(\bar{u}_{0}\right)$, который может оказаться как положительным, так и отрицательным. Если не накладывать дополнительных условий, то это обстоятельство, вообще говоря, не позволит обосновать построенную асимптотику решения.

Таким образом, в результате реализации метода угловых пограничных функций для исследования задачи (3)-(5) мы перешли к задачам (21)-(22). Дальнейшие исследования предполагают разрешение по крайней мере трех основных проблем: 
1. Имеет ли задача (21), (22) решение, удовлетворяющее экспоненциальной оценке убывания вида $(25)$ ?

2. Если задача (21), (22) имеет решение, удовлетворяющее экспоненциальной оценке вида (25), то имеют ли задачи (23), (24) решения, удовлетворяющие подобным оценкам?

3. Если задачи (21)-(24) разрешимы, т.е. если ряд (9) может быть построен, то имеет ли задача (3)-(5) решение, для которого этот ряд будет асимптотическим представлением при $\varepsilon \rightarrow 0$ в замкнутом прямоугольнике $\bar{\Omega}$ ?

Задачи для угловых пограничных функций $P_{k}^{*}\left(\xi_{*}, \tau\right), k \geqslant 0$, ставятся аналогично.

5. Монотонный случай. Рассмотрим случай, когда выполнено следующее дополнительное условие:

VI. Функция $F$ в угловых точках $(0,0)$ и $(1,0)$ прямоугольника $\Omega$ является квадратичной и монотонной на промежутке от корня $\bar{u}_{0}$ вырожденного уравнения до граничного значения $\phi$.

Справедливы следующие утверждения.

Теорема 1. Если выполнены условия I-VI, то задача (21), $(22)$ имеет решение $P_{0}(\xi, \tau)$ с оценкой вида (25).

Для доказательства существования решения этой задачи используется метод верхних и нижних решений (см. [8-10]), который заключается в том, что задача

$$
\begin{cases}L(Z)=0 & \text { в области } \Omega, \\ Z=h & \text { на границе } \partial \Omega\end{cases}
$$

имеет решение $Z$ в промежутке между барьерными функциями $Z_{-} \leqslant Z \leqslant Z_{+}$, если в области $\Omega$ выполняются неравенства

$$
L\left(Z_{+}\right) \leqslant 0, \quad L\left(Z_{-}\right) \geqslant 0, \quad Z_{-} \leqslant Z_{+},
$$

а на ее границе -

$$
Z_{-} \leqslant h \leqslant Z_{+} \text {. }
$$

В зависимости от вида функция $F$ строятся верхние и нижние решения задачи $(21),(22)$.

Теорема 2. Если выполнены условия I-VI, то задачи (23), (24) имеют решения $P_{k}(\xi, \tau)$ с оценками вида (25).

Барьерные функции для задачи $(21),(22)$ строятся с расчетом, чтобы коэффициент $F_{u}^{\prime}\left(\bar{u}_{0}\right)$ в задачах $(23),(24)$ был положителен. Это гарантирует существование решений $P_{k}(\xi, \tau)$ с оценками вида (25). Общий монотонный случай рассмотрен в работе [6].

6. Немонотонный случай. От функции $F$ не будем требовать монотонности на промежутке от корня $\bar{u}_{0}$ вырожденного уравнения до граничного значения $\phi$. Предположим, что нелинейная задача, определяющая главный член угловой части асимптотики, разрешима. В силу условия III и экспоненциальных оценок убывания для пограничных функций найдется такое неотрицательное число $\rho_{0}$, что в области

$$
\Omega_{0}=\left\{(\xi, \tau) \mid \xi \geqslant \rho_{0}, \tau \geqslant \rho_{0}\right\}
$$

значения производной на нулевом приближении удовлетворяют неравенству

$$
F_{u}^{\prime}\left(\bar{u}_{0}+\Pi_{0}+Q_{0}+P_{0}\right) \geqslant m^{2},
$$

где $m$ - некоторое положительное число. Однако в приграничных полосах

$$
\Omega_{1}=\left\{(\xi, \tau) \mid \xi \geqslant \tau, 0 \leqslant \tau \leqslant \rho_{0}\right\}, \quad \Omega_{2}=\left\{(\xi, \tau) \mid 0 \leqslant \xi \leqslant \rho_{0}, \tau \geqslant \xi\right\}
$$

производная может принимать отрицательные значения. Поэтому задачи (23), (24) не всегда будут иметь решения, удовлетворяющие оценкам вида (25). В связи с этим введем дополнительные условия.

$\mathbf{V I I}_{+}$. Задача $(21),(22)$ имеет решение $P_{0}(\xi, \tau)$ с оценкой вида $(25)$, и во всем квадранте $\mathbb{R}_{+}^{2}$ значения производной $F_{u}^{\prime}$ на нулевом приближении удовлетворяют неравенству (26). 
Если условие $\mathrm{VII}_{+}$не выполняется, то в приграничных полосах $\Omega_{1}$ и $\Omega_{2}$ производная $F_{u}^{\prime}$ может принимать отрицательные значения. Вместо $\mathrm{VII}_{+}$потребуем выполнения другого условия.

VII_. Задача $(21),(22)$ имеет решение $P_{0}(\xi, \tau)$ с оценкой вида $(25)$, и в приграничных полосах $\Omega_{1}$ и $\Omega_{2}$ квадранта $\mathbb{R}_{+}^{2}$ производная $F_{u}^{\prime}$ на нулевом приближении принимает отрицательные значения, но при этом

$$
F_{u}^{\prime}\left(\bar{u}_{0}+\Pi_{0}+Q_{0}+P_{0}\right) \geqslant-q^{2}
$$

где число $q \in\left(0, q_{0}\right)$, а $q_{0}$ является решением уравнения

$$
q_{0}^{2}-\frac{\pi}{2 \rho_{0}} \operatorname{ctg} \frac{\rho_{0} q_{0}}{a}=0 \text { на промежутке } q_{0} \in\left(0, \frac{\pi a}{2 \rho_{0}}\right) .
$$

Вместе условия VII + и VII_ образуют условие VII. В [7] установлен следующий результат.

Теорема 3. Если выполнены условия I-V и VII, то задачи (23), (24) имеют решения $P_{k}(\xi, \tau)$ с оченками вида (25).

При условии $\mathrm{VII}_{+}$верхние и нижние решения задач (23), (24) строятся во всем квадранте. При условии VII_ верхние и нижние решения задач (23), (24) строятся отдельно в каждой из трех подобластей, затем эти куски гладко стыкуются друг с другом.

7. Оценка остаточного члена. При условиях I-V плюс VI или VII ряд (9) оказывается полностью построенным.

Теорема 4. Если выполнены условия I-V плюс VI или VII, то для достаточно малых в задача (3)-(5) имеет решение $u(x, t, \varepsilon)$, для которого ряд

$$
\sum_{k=0}^{\infty} \varepsilon^{k}\left(\bar{u}_{k}(x, t)+\Pi_{k}(x, \tau)+Q_{k}(\xi, t)+Q_{k}^{*}\left(\xi_{*}, t\right)+P_{k}(\xi, \tau)+P_{k}^{*}\left(\xi_{*}, \tau\right)\right)
$$

является асимптотическим представлением при $\varepsilon \rightarrow 0$ в замкнутом прямоугольнике $\bar{\Omega}$.

Доказательство теоремы основано на разрешимости задач для пограничных функций $\Pi_{k}, Q_{k}$, $Q_{k}^{*}, P_{k}$ и $P_{k}^{*}$ при $k \geqslant 1$ и приведено в [5].

\section{СПИСОК ЛИТЕРАТУРЫ}

1. Бутузов В. Ф. Асимптотика решения разностного уравнения с малыми шагами в прямоугольной области// Ж. вычисл. мат. мат. физ. - 1972. - 3. - С. 582-597.

2. Васильева А. Б., Бутузов В. Ф. Асимптотические методы в теории сингулярных возмущений. - М.: Высшая школа, 1990.

3. Вишик М. И., Люстерник Л. А. Регулярное вырождение и пограничный слой для линейных дифференциальных уравнений с малым параметром// Усп. мат. наук. - 1957. - 5. - С. 3-122.

4. Денисов И. В. Первая краевая задача для квазилинейного сингулярно возмущенного параболического уравнения в прямоугольнике// Ж. вычисл. мат. мат. физ. - 1996. - 10. - С. 56-72.

5. Денисов И. В. Угловой пограничный слой в краевых задачах для сингулярно возмущенных параболических уравнений с квадратичной нелинейностью// Ж. вычисл. мат. мат. физ. $-2017 .-2 .-$ C. $255-274$.

6. Денисов И. В. Угловой пограничный слой в краевых задачах для сингулярно возмущенных параболических уравнений с монотонной нелинейностью// Ж. вычисл. мат. мат. физ. - 2018. - 4. - С. 1-11.

7. Денисов И. В. Угловой пограничный слой в краевых задачах для сингулярно возмущенных параболических уравнений с нелинейностями// Ж. вычисл. мат. мат. физ. - 2019. - 1. - С. 102-117.

8. Amann $H$. On the existence of positive solutions of nonlinear elliptic boundary-value problems// Indiana Univ. Math. J. - 1971. - 2. - P. 125-125.

9. Amann H. Periodic solutions of semilinear parabolic equations// in: Nonlinear Analysis: Collections of Papers in Honor of Erich Rothe. - New York: Academic Press, 1978. — P. 1-29. 
10. Sattinger D. H. Monotone methods in nonlinear elliptic and parabolic boundary-value problems// Indiana Univ. Math. J. - 1972. — 11. - P. 979-1000.

Денисов Игорь Васильевич

Тульский государственный педагогический университет им. Л. Н. Толстого

E-mail: den_tspu@mail.ru

Денисов Алексей Игоревич

Национальный исследовательский университет «Высшая школа экономики», Москва 